\title{
Parallel enumeration of degree sequences of simple graphs II
}

\author{
Antal IVÁNYI \\ Eötvös Loránd University, \\ Faculty of Informatics \\ Budapest \\ email: tony@onf .elte.hu

\section{Gergő GOMBOS} \\ Eötvös Loránd University, \\ Faculty of Informatics \\ Budapest \\ email: ggombos@inf .elte.hu
}

\section{Loránd LUCZ}

Eötvös Loránd University,

Faculty of Informatics

Budapest

email: lorand.lucz@gmail.com

\section{Tamás MATUSZKA \\ Eötvös Loránd University, Faculty of Informatics Budapest}

email: matuszka1987@gmail.com

\begin{abstract}
In the paper we report on the parallel enumeration of the degree sequences (their number is denoted by $G(n)$ ) and zerofree degree sequences (their number is denoted by $\left(G_{z}(n)\right.$ ) of simple graphs on $n=30$ and $n=31$ vertices. Among others we obtained that the number of zerofree degree sequences of graphs on $n=30$ vertices is $\mathrm{G}_{z}(30)=5876236938019300$ and on $\mathrm{n}=31$ vertices is $\mathrm{G}_{z}(31)=$ 22974847474172374 . Due to Corollary 21 in [52] these results give the number of degree sequences of simple graphs on 30 and 31 vertices.
\end{abstract}

\section{Introduction}

In the practice an often occuring problem is the ranking of different objects (examples can be found e.g. in [52]), assigning points to the objects and then ranking of the objects on the base of the sum of the assigned to them points.

Computing Classification System 1998: G.2.2.

Mathematics Subject Classification 2010: 05C85, 68R10

Key words and phrases: simple directed graphs, linear Erdős-Gallai and Havel-Hakimi algorithm, enumeration of graphical sequences 
Especially extensive bibliography has the case when the results are represented by a simple graph and the problem is the test, reconstruction and enumeration of the degree sequences. Havel in 1955 [42], Erdös and Gallai in 1960 [16, 32, 77], Hakimi in 1962 [39], Knuth in 2008 [61], Tripathi et al. in 2010 [89] proposed a method to decide, whether a sequence of nonnegative integers can be the degree sequence of a simple graph. Sierksma and Hoogeven in 1991 [83] compared seven known methods. The running time of their algorithms in worst case is $\Omega\left(\mathrm{n}^{2}\right)$. In 2007 Takahashi [86], in 2009 Hell and Kirkpatrick [43], in 2011 Iványi et al. [52] and in April of 2012 Király [58] proposed an algorithm, whose worst running time is $\Theta(n)$.

There are several new proofs for the classical Havel-Hakimi and Erdős-Gallai theorems $[26,32,63,70,75,87,88,89]$.

Extensions of the algorithms for (0,b)-graphs [8, 9, 24, 23, 25, 27, 69, 75, $90,92]$ and ( $\mathrm{a}, \mathrm{b})$-graphs $[44,45,46,53]$ are also known.

As an application of our linear time algorithm we describe ERDős-GALLAIENUMERATIVE algorithm (EGE) and its parallel version used to enumerate the different degree sequences of simple graphs for 30 vertices. We also present the linear test version of Havel-Hakimi algorithm (HHL).

Let $n \geq 1$. We call a sequence $\mathbf{s}=\left(s_{1}, \ldots, s_{n}\right)(l, u, n)$-bounded, if $0 \leq$ $s_{i} \leq n$ for $i=1, \ldots, n, n$-bounded, if it is $(0, n-1, n)$-bounded, $n$-regular, if the conditions $n-1 \geq s_{1} \geq \cdots \geq s_{n} \geq 0$ hold, and $n$-even, if the sum of the elements of $\mathbf{s}$ is even. If there exists a graph with $\mathfrak{n}$ vertices which has the degree sequence $\mathbf{s}$, then we say that $\mathbf{s}$ is $\mathbf{n}$-graphical. If such graph does not exist, then we say that $\mathbf{s}$ is nongraphical. A sequence is zerofree, if it does not contain zero. If $\boldsymbol{n}$ is not necessary, then we omit it in the terms $n$-bounded, $n$-regular, $n$-even and $n$-graphical. The first $i$ elements of an $n$-regular $\mathbf{s}$ are called the head, and the last $n-i$ elements are called the tail, belonging to the element $i$ of $\mathbf{s}$.

\section{Earlier results}

A classical problem of the graph theory is the enumeration of the sorted degree sequences of different graphs - among others simple graphs. For example The On-Line Encyclopedia of Integer Sequences contains for $\mathrm{n}=1, \ldots, 29$ vertices the number of degree sequences of simple graphs (the values for $n=20, \ldots, 23$ were set in July of 2011 by Nathann Cohen [28], and for 24, .., 29 in 15 November, 2011 by us $[48,52])$ and the number of zerofree degree sequences of simple graphs (the values for $n=1, \ldots, 9$ were set in 12 June, 2004 by 
N. J. Sloane, for $n=10, \ldots, 20$ in 12 August, 2006 by Gordon Royle, for $\mathrm{n}=21$, 22, and 23 in August 31, 2011, and in December 10, 2012 by Frank Ruskey [80], and the values for $n=24, \ldots, 29$ by us $[50,51]$.

In this section we review the theoretical and practical results connected with the enumeration of simple graphs.

\subsection{Exact enumeration results}

It is known [52, equation (23)] that if $n \geq 1$, then the number $R(n)$ of the regular sequences is

$$
R(n)=\left(\begin{array}{c}
2 n-1 \\
n
\end{array}\right)
$$

and the number $R_{z}(n)$ of the zerofree regular sequences is [52, equation (24)]

$$
R_{z}(n)=\left(\begin{array}{c}
2 n-2 \\
n
\end{array}\right)
$$

implying [52]

$$
\lim _{n \rightarrow \infty} \frac{R(n+1)}{R(n)}=\lim _{n \rightarrow \infty} \frac{R_{z}(n+1)}{R_{z}(n)}=4
$$

and

$$
\lim _{n \rightarrow \infty} \frac{R_{z}(n)}{R(n)}=\frac{1}{2}
$$

and

$$
R(n)=\frac{4^{n}}{2 \sqrt{\pi n}}+O\left(\frac{4^{n}}{n^{3 / 2}}\right)
$$

and

$$
R_{z}(n)=\frac{4^{n}}{4 \sqrt{\pi n}}+O\left(\frac{4^{n}}{n^{3 / 2}}\right) .
$$

Table 1 in [52] shows the values values of $R(n)$ for $n=1, \ldots, 38$, Table 4 in [51] for $n=39, \ldots, 60$, and in $[47,51,68]$ the values are presented for $n=1, \ldots, 1200$. Table 1 in Subsection 3.3 presents the values $R(n) / R(n+1)$ for $n=1, \ldots, 32$ and [68] for $n=1, \ldots, 1200$.

Figure 1 in Subsection 3.3 shows the values of $\left.R_{z}(n) / R_{z}(n+1)\right)$ for $n=$ $1, \ldots, 32$.

In 1987 Ascher derived the following explicit formula for the number $E(n)$ of even sequences. 
Lemma 1 (Ascher [2], Sloane and Pfoffe [85]) If $\mathfrak{n} \geq 1$, then the number of even sequences $\mathrm{E}(\mathrm{n})$ is

$$
E(n)=\frac{1}{2}\left(\left(\begin{array}{c}
2 n-1 \\
n
\end{array}\right)+\left(\begin{array}{l}
n-1 \\
\lfloor n / 2\rfloor
\end{array}\right)\right)
$$

Proof. See [2].

Table 1 in [52] contains the values of $E(n)$ and $E(n+1) / E(n)$ for $n=$ $1, \ldots, 31$.

(7) implies (see [52])

$$
\lim _{n \rightarrow \infty} \frac{E(n+1)}{E(n)}=4
$$

and

$$
E(n)=\frac{4^{n}}{8 \sqrt{\pi n}}+O\left(\frac{4^{n}}{n^{3 / 2}}\right)
$$

further (1) and (7) imply

$$
\lim _{n \rightarrow \infty} \frac{E(n)}{R(n)}=\frac{1}{2}
$$

(2) and (7) imply

$$
\frac{R_{z}(n)}{E(n)}=\frac{2 n-2}{2 n-1}=1-\frac{1}{2 n-1} \text { and } \lim _{n \rightarrow \infty} \frac{R_{z}(n)}{E(n)}=1 .
$$

Table 1 in [52] shows the values of $E(n)$ for $n=1, \ldots$, 38, Table 4 in [51] for $n=39, \ldots, 60$, the list of [64] for $n=1, \ldots, 1000$, and [68] for $\mathrm{n}=31, \ldots, 1200$.

Figure 3 in [52] shows the values of $E_{z}(n)$ for $n=1, \ldots, 20$, and [68] $n=$ $1, \ldots, 1200$. Table 5 in $[51]$ shows the values of $E_{z}(n / R(n)$ for $n=1, \ldots, 20$.

Using (1) and (7) we computed $E(n)$ and $E(n+1) / E(n)$ for $i=1, \ldots, 750$ (see $[52,68])$. Recently Librandi [64] published the values of $E(n)$ up to $n=$ 1000 and we continued the computations up to 1200 [51, 68].

The following theorem gives a very useful connection between the values of $G(n)$ and $G_{z}(n)$ : it helped to decrease the computing time of $G(29)$ with about $50 \%$.

Lemma 2 (Iványi, Lucz, Móri, Sótér [52]) If $\mathrm{n} \geq 2$, then the number of $\mathrm{n}$ graphical sequences $\mathrm{G}(\mathrm{n})$ can be computed from the number of $(\mathrm{n}-1)$-graphical sequences $\mathrm{G}(\mathrm{n}-1)$ and the number of $\mathrm{n}$-graphical zero-free sequences $\mathrm{G}_{z}$ :

$$
G(n)=G(n-1)+G_{z}(n),
$$


and if $\mathrm{n} \geq 1$ then

$$
G(n)=1+\sum_{i=2}^{n} G_{z}(i) .
$$

Proof. If an even sequence $s=\left(s_{1}, \ldots, s_{n}\right)$ contains at least one zero, then $s_{n}=0$ and $s^{\prime}=\left(s_{1}, \ldots, s_{n-1}\right)$ is graphical or not. If $\mathbf{a}=\left(a_{1}, \ldots, a_{n-1}\right)$ is $(n-1)$-graphical, then $\mathbf{a}^{\prime}=\left(a_{1}, \ldots, a_{n-1}, 0\right)$ is $n$-graphical.

The set of the $n$-graphical sequences $\mathcal{S}(n)$ consists of two subsets: set of zerofree sequences $\mathcal{S}_{z}(\mathrm{n})$ and the set of the remaining sequences $\mathcal{S}_{0}(\mathrm{n})$. There is a bijection between the set of the $(n-1)$-graphical sequences and such $n$ graphical sequences, which contain at least one zero. Therefore $|\mathcal{S}|=\left|\mathcal{S}_{z}\right|+$ $\left|\mathcal{S}_{0}\right|=\mathrm{G}_{z}(\mathrm{n})+\mathrm{G}(\mathrm{n}-1)$.

Using the parallel version EGP (see the next section) of EGE we computed $\mathrm{G}(\mathrm{n})$ up to $\mathrm{n}=29$. These numbers can be found in Table 2 of [52].

Theorem 3 (Burns [22]) There exist positive constants $\mathrm{c}$ and $\mathrm{C}$ such that the following bounds of the function $\mathrm{G}(\mathrm{n})$ are true for $\mathrm{n} \geq 1$ :

$$
\frac{4^{n}}{c n}<G(n)<\frac{4^{n}}{(\log n)^{C} \sqrt{n}} .
$$

Proof. See [22].

This result implies that the asymptotic density of the graphical sequences is zero among the even sequences.

Corollary 4 If $\mathrm{n} \geq 1$, then there exists a positive constant $\mathrm{C}$ such that

$$
\frac{G(n)}{E(n)}<\frac{1}{\left(\log _{2} n\right)^{C}}
$$

and

$$
\lim _{n \rightarrow \infty} \frac{G(n)}{E(n)}=0
$$

Proof. (13) is a direct consequence of (7) and (12).

Table 1 in [52] contains the values of $G(n)$ and $G(n+1) / G(n)$ for $n=$ $1, \ldots, 29$. Table 5 in $[51]$ contains values of $G_{z}(n), G_{z}(n) / R(n)$, and $G(n) / R(n)$ for $n=1, \ldots, 29$.

We remark that a zerofree degree sequence belongs to a graph not containing isolated vertex, therefore the number of zerofree graphical degree sequences 
$\mathrm{G}_{z}(\mathrm{n})$ is at the same time also the number of degree sequences of simple graphs, not containing isolated vertex.

There are several classic asymptotic results, e.g. due to Bender and Canfield [7], Bollobás [17, 18, 19], Harary and Palmer [41], Kleitman and Winston [56, 60], Reid [78], Winston and Kleitman [91]. A modern direction is to get approximate results by sampling of random graphs (see e.g. the papers of Erdős, Király and Miklós [34], further of Miklós, Erdős and Soukup [?].

An interesting connected problem is the characterization of pairs of different directed graphs having a pair of prescribed indegree and outdegree sequences $[8,9,10,11,12,14,15,20,40,72,76,81]$.

Another interesting related questions are the unicity of the realizations of the degree sequences $[29,55,62,82]$ and the parallel realization of degree sequences [1].

Several recent papers consider the problem of approximate enumeration of the number of all realizations of simple graphs (see e.g. [13, 34, 35, 36, 37, 38, 59, 71]). In 1978 Bender and Canfield [7] characterized the asymptotic number of realizations of given graphical degree sequences, while in 2012 Zoltán Király [58] proposed an algorithm which with polynomial delay lists all realizations of a given graphical sequence.

\subsection{Earlier algorithmic results}

In this subsection the linear Havel-Hakimi algorithm (HHL) based on HavelHakimi theorem [39, 42] and the enumerating Erdös-Gallai algorithm (EGE) based on Erdős-Gallai theorem [32] are shortly described.

\subsubsection{Linear Havel-Hakimi algorithm (HHL)}

In a previous paper [52] we described the classical Havel-Hakimi [39, 42] and Erdős-Gallai [32] algorithms and their some improvements as linear ErdősGallai (EGL) and jumping Erdős-Gallai (EGLJ) algorithms.

it is worth to remark that our linear Erdős-Gallai algorithm is applied in the solution of different problems connected with degree sequences [5, 6, 21, 31].

Here we present the linear version of Havel-Hakimi algorithm (HHL) [46] and compare it with the previous linear algorithms EGL and EGLJ [52]. It is important to remark that this linear version of $\mathrm{HH}$ only tests the investigated sequences without their reconstruction.

In the worst case the original Havel-Hakimi algorithm requires quadratic time to test the $(0,1, n)$-regular sequences. Using the new concepts weight 
point and reserve we reduced the worst running time to $O(n)$.

Let $s=\left(s_{1}, \ldots, s_{n}\right)$ be a potential graphical sequence. The definition of the weight point $w_{i}$ belonging to $s_{i}$ was introduced in [52] in connection with ERDÖS-GALLAI-LinEAR: if $s_{1} \geq i$, then $w_{i}$ is the largest $k(1 \leq k \leq n)$ having the property $s_{k} \geq i$. But if $s_{1}<i$, then $w_{i}=0$. EGL exploits the property $w_{i}$ ensuring that if $i \leq \mathcal{w}_{i}$, then the key expression min $j, s_{k}$ in the Erdös-Gallai theorem equals $i$, otherwise equals $s_{k}$.

In HHL the weight point $w_{i}$ determines the increment of the tail capacity when we switch to the investigation of the next element of $s$.

The reserve $r_{i}$ belonging to $s_{i}$ is defined as the unused part of the actual tail capacity and can be computed by the formulas

$$
r_{1}=w_{1}-1-s_{1}
$$

and

$$
r_{i}=w_{i}+r_{i-1}-s_{i} \quad \text { for } \quad 2 \leq i \leq n-1 .
$$

Theorem 5 The running time of HAVEL-HAKIMI-LINEAR is in best case $\Theta(1)$, and in worst case it is $\Theta(\mathrm{n})$.

Proof. If the condition in line 1 or 3 holds, then the running time is $\Theta(1)$. If not, then we decrease the actual $w$ at most $n$ times and the remaining operations require $\mathrm{O}(1)$ operations for all reductions.

\subsubsection{Enumerating Erdős-Gallai algorithm (EGE)}

A classical problem of the graph theory is the enumeration of the degree sequences of different graphs - among others simple graphs. For example The On-Line Encyclopedia of Integer Sequences [84] contains for $\mathrm{n}=1, \ldots, 30$ vertices the number of degree sequences of simple graphs (the values for $n=$ 20, .., 23 were set in July of 2011 by Nathann Cohen, in November 15, 2011 for $24, \ldots, 29$ and in 29 July of 2013 for $n=30$ by us [48]).

We applied the new quick EGL to get these numbers for larger values of $n$.

Our starting point was to test all regular sequences and so to enumerate the graphical ones. Equation 1 gives the number of regular sequences.

According to Erdős-Gallai theorem [32] the sum of the elements of a graphical sequence is always even. Therefore it is sufficient to test only the even sequences. In 1987 Ascher [2] derived Lemma 1, containing an explicit formula for the number of even sequences $E(n)$.

According to Lemma 2 it is enough to test only the zerofree even sequences. 
This lemma was the base of Erdős-Gallai Enumerative algorithm (EGE) used to enumerate the graphical sequences for $n=23, \ldots, 29$ [51].

We enumerated the graphical sequences of simple graphs on $n=30$ and 31 vertices using algorithm EGE2. The running time of EGE was substantially (with about $30 \%$ ) decreased due to Lemma 9.

We prepare the enumeration of degree sequences of simple graphs on 32 vertices. The running time of EGE2 would be about 320 years for a computer with one processor having $2,2 \mathrm{GHz}$ speed. We wish to decrease the running time of EGE2 using Lemmas 10 and 11.

\subsection{Earlier simulation results}

The papers $[44,45,46,51,52,66]$ and OEIS $[64,73,74]$ contain many simulation results. We describe them together with the new results in Subsection 3.3 .

It is worth to mention other methods of enumeration of graph sequences as generation of random graphs (e.g. [65] and generation of graphical partitions (see e.g. [3, 4, 30, 33].

\section{New results}

In this section we describe the new mathematical and simulation results.

\subsection{New enumerative results}

At first we give a new formula for the number of zerofree even sequences. This formula is more sophisticated than Ascher's formula, and its application requires more time, but it has the adventage that we can extend it to a formula for $E_{z}(n)$. Let $\mathbf{s}$ be an $n$-even sequence and let $\mathbf{s}^{\prime}=\left(s_{1}^{\prime}, \ldots, s_{n}^{\prime}\right)$ be defined by $s_{i}^{\prime}=s_{i}+n-i$ for $i=1, \ldots, n$. Then the number of different possible sequences $\mathbf{s}$ is $E(n)$ and the number of different sequences $\mathbf{s}^{\prime}$ is $R(n)$.

If $j=0,1,2$, or 3 and $n=4 k+j$, then let $E(n)$ be denoted by $E(k, j)$.

Lemma 6 If $n \geq 1$ and $n=4 k+j$, then

$$
\begin{aligned}
& E(k, 0)=\sum_{i=0}^{2 k-1}\left(\begin{array}{c}
4 k-1 \\
2 i
\end{array}\right)\left(\begin{array}{c}
4 k \\
4 k-2 i
\end{array}\right), \\
& E(k, 1)=\sum_{i=0}^{2 k}\left(\begin{array}{c}
4 k \\
2 i
\end{array}\right)\left(\begin{array}{c}
4 k+1 \\
4 k-2 i+1
\end{array}\right),
\end{aligned}
$$




$$
\begin{aligned}
& E(k, 2)=\sum_{i=0}^{2 k}\left(\begin{array}{c}
4 k+1 \\
2 i+1
\end{array}\right)\left(\begin{array}{c}
4 k+2 \\
4 k-2 i+1
\end{array}\right), \\
& E(k, 3)=\sum_{i=0}^{k}\left(\begin{array}{c}
4 k+2 \\
2 i+1
\end{array}\right)\left(\begin{array}{c}
4 k+3 \\
4 k-2 i+2
\end{array}\right) .
\end{aligned}
$$

Proof. Let

$$
\sum_{i=1}^{n} s_{i}=S(\mathbf{s}) \text { and } \sum_{i=1}^{n} s_{i}^{\prime}=S^{\prime}(\mathbf{s}) .
$$

According to the value of $j$ we consider four cases. Since $\mathbf{s}$ is an even sequence, therefore $S(\mathbf{s})$ is even in all cases.

1. If $j=0$, then

$$
S^{\prime}(\mathbf{s})=S(\mathbf{s})+\sum_{i=0}^{4 k-1} i=S(\mathbf{s})+2 k(4 k-1),
$$

and so $\mathbf{S}\left(\mathbf{s}^{\prime}\right)$ is also even, therefore it contains an even number of odd elements. The interval $[0,8 k-2]$ contains $8 k-1$ elements and among them $4 k$ even and $4 k-1$ odd elements, so for $s^{\prime}$ we can choose $2 i$ odd elements from $4 k-1$ candidates and $4 k-2 i(i=0,1, \ldots, 2 k-1)$ even elements from $4 k+1$ candidates, so

$$
E(k, 0)=\sum_{i=0}^{2 k-1}\left(\begin{array}{c}
4 k-1 \\
2 i
\end{array}\right)\left(\begin{array}{c}
4 k \\
4 k-2 i
\end{array}\right) .
$$

2. If $j=1,2$ or $j=3$, then the proof is similar to the proof in the first case.

For example let $\mathfrak{n}=4$, then $k=1, j=0$ and

$$
E(4)=E(1,0)=\sum_{i=0}^{1}\left(\begin{array}{c}
3 \\
2 i
\end{array}\right)\left(\begin{array}{c}
4 \\
4-2 i
\end{array}\right)=1 \cdot 1+3 \cdot 6=19 .
$$

As another example let $n=6$, then $k=1, j=2$ and

$$
E(6)=\sum_{i=0}^{2}\left(\begin{array}{l}
5 \\
1
\end{array}\right)\left(\begin{array}{l}
6 \\
3
\end{array}\right)+\left(\begin{array}{l}
5 \\
5
\end{array}\right)\left(\begin{array}{l}
6 \\
1
\end{array}\right)=530+200+6=236
$$


Let the number of zerofree even sequences denoted by $E_{z}(n)$. Let $\mathbf{q}=$ $\left(q_{1}, \ldots, q_{n}\right)$ be a zerofree $n$-even sequence and let $\mathbf{q}^{\prime}=\left(q_{1}^{\prime}, \ldots, q_{n}^{\prime}\right)$ be defined by $q_{i}^{\prime}=q_{i}+n-i$ for $i=1, \ldots, n$. Then the number of different possible sequences $\mathbf{q}$ is $E_{z}(n)$ and the number of different sequences $\mathbf{q}^{\prime}$ is $R_{z}(n)$.

Theorem 7 Let $\mathrm{n}=4 \mathrm{k}+\mathrm{j}$ for $\mathrm{k}=0,1, \ldots$ and $\mathrm{j}=0,1,2,3$, further let $\mathrm{E}_{z}(\mathrm{n})$ be denoted by $\mathrm{E}_{z}(k, j)$. Then

$$
\begin{gathered}
E_{z}(k, 0)=\sum_{i=0}^{2 k-1}\left(\begin{array}{c}
4 k-1 \\
2 i
\end{array}\right)\left(\begin{array}{c}
4 k-1 \\
4 k-2 i
\end{array}\right), \\
E_{z}(k, 1)=\sum_{i=0}^{2 k}\left(\begin{array}{c}
4 k \\
2 i
\end{array}\right)\left(\begin{array}{c}
4 k \\
4 k-2 i+1
\end{array}\right), \\
E_{z}(k, 2)=\sum_{i=0}^{2 k}\left(\begin{array}{c}
4 k+1 \\
2 i+1
\end{array}\right)\left(\begin{array}{c}
4 k+1 \\
4 k-2 i+1
\end{array}\right), \\
E_{z}(k, 3)=\sum_{i=0}^{2 k+1}\left(\begin{array}{c}
4 k+2 \\
2 i+1
\end{array}\right)\left(\begin{array}{c}
4 k+2 \\
4 k-2 i+2
\end{array}\right) .
\end{gathered}
$$

Proof. Let

$$
\sum_{i=1}^{n} q_{i}=Q(q) \text { and } \sum_{i=1}^{n} q_{i}^{\prime}=Q^{\prime}(\mathbf{q}) .
$$

According to the value of $\boldsymbol{j}$ we consider four cases. Since $\mathbf{q}$ is an even sequence, therefore $\mathrm{Q}(\mathbf{q})$ is alwys even.

1. If $j=0$, then

$$
\mathrm{Q}^{\prime}(\mathbf{q})=\mathrm{Q}(\mathbf{q})+\sum_{i=0}^{4 k-1} i=\mathrm{Q}(\mathbf{q})+2 \mathrm{k}(4 \mathrm{k}-1)
$$

is even, therefore the number of odd elements of $\mathbf{q}^{\prime}$ is also even. The interval $[1,8 k-2]$ contains $8 k-2$ elements and among them $4 k-1$ even and $4 k-1$ odd elements, so for $\mathbf{q}^{\prime}$ we can choose $2 i$ odd elements from $4 k-1$ candidates and $4 k-2 i(i=0, \ldots, 2 k-1)$ even elements from $4 k-1$ candidates, so we get $(26)$. 
2. If $j=1$, then

$$
\mathrm{Q}^{\prime}(\mathbf{q})=\mathrm{Q}(\mathbf{q})+\sum_{i=0}^{4 \mathrm{k}} i=\mathrm{Q}(\mathbf{q})+2 \mathrm{k}(4 \mathrm{k}+1),
$$

is even, therefore the number of odd elements of $\mathbf{q}^{\prime}$ is also even. The interval $[1,8 k]$ contains $8 k$ elements and among them $4 k$ odd and $4 k$ even elements, so for $\mathbf{q}^{\prime}$ we can choose $2 i$ odd elements from $4 k$ candidates and $4 k-2 i+1(i=0, \ldots, 2 k)$ even elements from $4 k-1$ candidates, so we get $(27)$.

3. If $j=2$, then

$$
\mathrm{Q}^{\prime}(\mathbf{q})=\mathrm{Q}(\mathbf{q})+\sum_{i=0}^{4 k+1} i=\mathrm{Q}(\mathbf{q})+(2 \mathrm{k}+1)(4 \mathrm{k}+1)
$$

is odd, therefore the number of odd elements of $\mathbf{q}^{\prime}$ is also odd. The interval $[1,8 k+2]$ contains $8 k+2$ elements and among them $4 k+1$ even and $4 k+1$ odd elements, so for $\mathbf{q}^{\prime}$ we can choose $2 i+1$ odd elements from $4 k+2$ candidates and $4 k-2 i-1(i=0, \ldots, 2 k-1)$ even elements from $4 k+1$ candidates, so we get $(28)$.

4. If $j=3$, then

$$
\mathrm{Q}^{\prime}(\mathbf{q})=\mathrm{Q}(\mathbf{q})+\sum_{i=0}^{4 k+2} i=\mathrm{Q}(\mathbf{q})+(2 \mathrm{k}+1)(4 \mathrm{k}+3),
$$

and so $\mathrm{Q}\left(\mathbf{q}^{\prime}\right)$ is also odd, therefore $\mathbf{q}^{\prime}$ contains an odd number of odd elements. The interval $[1,8 k+4]$ contains $8 k+4$ elements and among them $4 k+2$ even and $4 k+2$ odd elements, so for $\mathbf{q}^{\prime}$ we can choose $2 i+1$ odd elements from $4 k+2$ candidates and $4 k-2 i-1(i=0, \ldots, 2 k-1)$ even elements from $4 k+2$ candidates, so

$$
E_{z}(k, 3)=\sum_{i=0}^{2 k+1}\left(\begin{array}{c}
4 k+2 \\
2 i+1
\end{array}\right)\left(\begin{array}{c}
4 k+2 \\
4 k-2 i+2
\end{array}\right) .
$$

Table 1 shows the values of $R(n) / R(n+1), R_{z}(n) / R_{z}(n+1), E(n) / R(n)$, $E(n) / E(n+1), E_{z}(n) / E_{z}(n+1)$, and $E_{z}(n) / R_{z}(n)$ for $n=1, \ldots, 32$. 


\begin{tabular}{|c|c|c|c|c|c|c|}
\hline$n$ & $\frac{R(n)}{R(n+1)}$ & $\frac{R_{z}(n)}{R_{z}(n+1)}$ & $\frac{E(n)}{R(n)}$ & $\frac{E(n)}{E(n+1)}$ & $\frac{E_{z}(n)}{E_{z}(n+1)}$ & $\frac{E_{z}(n)}{R_{z}(n)}$ \\
\hline 1 & 0.333333 & 0.000000 & 1.00000000 & 0.000000 & 0.000000 & --- \\
\hline 2 & 0.300000 & 0.250000 & 0.66666667 & 0.500000 & 0.500000 & 1.000000 \\
\hline 3 & 0.287714 & 0.266667 & 0.60000000 & 0.222220 & 0.222222 & 0.500000 \\
\hline 4 & 0.277778 & 0.257857 & 0.487179 & 0.321427 & 0.321429 & 0.600000 \\
\hline 5 & 0.270562 & 0.266667 & 0.523810 & 0.254545 & 0.254545 & 0.500000 \\
\hline 6 & 0.269231 & 0.265151 & 0.510823 & 0.277778 & 0.277778 & 0.523810 \\
\hline 7 & 0.266667 & 0.263736 & 0.505828 & 0.260698 & 0.260698 & 0.500000 \\
\hline 8 & 0.264706 & 0.262500 & 0.502720 & 0.265559 & 0.265559 & 0.505828 \\
\hline 9 & 0.263158 & 0.261437 & 0.501440 & 0.260687 & 0.260687 & 0.500000 \\
\hline 10 & 0.261905 & 0.260526 & 0.500682 & 0.261276 & 0.261276 & 0.501440 \\
\hline 11 & 0.260870 & 0.259740 & 0.500357 & 0.259555 & 0.259555 & 0.500000 \\
\hline 12 & 0.260000 & 0.259058 & 0.500171 & 0.259243 & 0.259243 & 0.500357 \\
\hline 13 & 0.259259 & 0.258461 & 0.500089 & 0.258415 & 0.258416 & 0.500000 \\
\hline 14 & 0.258621 & 0.257937 & 0.500043 & 0.257982 & 0.257982 & 0.500089 \\
\hline 15 & 0.258065 & 0.257471 & 0.500022 & 0.257460 & 0.257460 & 0.500000 \\
\hline 16 & 0.257578 & 0.257056 & 0.500011 & 0.257068 & 0.257068 & 0.500022 \\
\hline 17 & 0.257143 & 0.256684 & 0.500005 & 0.256682 & 0.256682 & 0.500000 \\
\hline 18 & 0.256757 & 0.256349 & 0.500003 & 0.256352 & 0.256352 & 0.500006 \\
\hline 19 & 0.256410 & 0.256046 & 0.500001 & 0.256045 & 0.256045 & 0.500000 \\
\hline 20 & 0.256098 & 0.255769 & 0.500001 & 0.255770 & 0.255770 & 0.500001 \\
\hline 21 & 0.255814 & 0.255517 & 0.50000034 & 0.255517 & 0.255517 & 0.500000 \\
\hline 22 & 0.255556 & 0.255285 & 0.50000016 & 0.255286 & 0.255286 & 0.50000034 \\
\hline 23 & 0.255319 & 0.255072 & 0.50000009 & 0.255072 & 0.255072 & 0.50000000 \\
\hline 24 & 0.255102 & 0.254876 & 0.50000004 & 0.254876 & 0.254876 & 0.50000000 \\
\hline 25 & 0.254902 & 0.254694 & 0.50000002 & 0.254694 & 0.254694 & 0.50000009 \\
\hline 26 & 0.254717 & 0.254525 & 0.50000001 & 0.254525 & 0.254525 & 0.50000000 \\
\hline 27 & 0.254545 & 0.254368 & 0.50000001 & 0.254368 & 0.254368 & 0.50000000 \\
\hline 28 & 0.254386 & 0.254221 & 0.50000000 & 0.254221 & 0.254221 & 0.50000000 \\
\hline 29 & 0.254237 & 0.254083 & 0.50000000 & 0.254083 & 0.254083 & 0.50000000 \\
\hline 30 & 0.254098 & 0.253854 & 0.50000000 & 0.253955 & 0.253955 & 0.50000000 \\
\hline 31 & 0.253968 & 0.253834 & 0.50000000 & 0.253834 & 0.253834 & 0.50000000 \\
\hline 32 & 0.253846 & 0.253720 & 0.50000000 & 0.253720 & 0.253720 & 0.50000000 \\
\hline
\end{tabular}

Table 1: The values of $R(n) / R(n+1), R_{z}(n) / R_{z}(n+1), E(n) / R(n), E(n) / E(n+$ $1), E_{z}(n) / E_{z}(n+1)$, and $E_{z}(n) / R_{z}(n)$ for $n=1, \ldots, 32$

It is remarkable that in $R(101) / R(102)$ and $R_{z}(101) / R_{z}(102)$ the first nine decimal digits are equal.

For example let $\mathrm{n}=4$, then $\mathrm{k}=1, \mathrm{j}=0$ and

$$
E_{z}(4)=E_{z}(1,0)=\left(\begin{array}{l}
3 \\
0
\end{array}\right)\left(\begin{array}{l}
3 \\
4
\end{array}\right)+\left(\begin{array}{l}
3 \\
2
\end{array}\right)\left(\begin{array}{l}
3 \\
2
\end{array}\right)=1 \cdot 0+3 \cdot 3=9 .
$$

If $n=5$, then $k=1, j=1$ and

$$
E_{z}(5)=\left(\begin{array}{l}
4 \\
0
\end{array}\right)\left(\begin{array}{l}
4 \\
5
\end{array}\right)+\left(\begin{array}{l}
4 \\
2
\end{array}\right)\left(\begin{array}{l}
4 \\
3
\end{array}\right)+\left(\begin{array}{l}
4 \\
4
\end{array}\right)\left(\begin{array}{l}
4 \\
1
\end{array}\right)=0+24+4=28 .
$$


If $\mathrm{n}=6$, then $\mathrm{k}=1, \mathrm{j}=2$ and

$$
E_{z}(6)=\left(\begin{array}{l}
5 \\
1
\end{array}\right)\left(\begin{array}{l}
5 \\
5
\end{array}\right)+\left(\begin{array}{l}
5 \\
3
\end{array}\right)\left(\begin{array}{l}
5 \\
3
\end{array}\right)+\left(\begin{array}{l}
5 \\
5
\end{array}\right)\left(\begin{array}{l}
5 \\
1
\end{array}\right)=5+100+5=110 .
$$

If $\mathrm{n}=7$, then $\mathrm{k}=1, \mathrm{j}=3$ and

$$
E_{z}(7)=\left(\begin{array}{l}
6 \\
1
\end{array}\right)\left(\begin{array}{l}
6 \\
6
\end{array}\right)+\left(\begin{array}{l}
6 \\
3
\end{array}\right)\left(\begin{array}{l}
6 \\
4
\end{array}\right)+\left(\begin{array}{l}
6 \\
5
\end{array}\right)\left(\begin{array}{l}
6 \\
2
\end{array}\right)=6+300+90=396 .
$$

If $n=8$, then $k=2, j=0$ and

$$
E_{z}(8)=\left(\begin{array}{l}
7 \\
0
\end{array}\right)\left(\begin{array}{l}
7 \\
8
\end{array}\right)+\left(\begin{array}{l}
7 \\
2
\end{array}\right)\left(\begin{array}{l}
7 \\
6
\end{array}\right)+\left(\begin{array}{l}
7 \\
4
\end{array}\right)\left(\begin{array}{l}
7 \\
4
\end{array}\right)+\left(\begin{array}{l}
7 \\
6
\end{array}\right)\left(\begin{array}{l}
7 \\
2
\end{array}\right)=1519 .
$$

Simulaton results in Table 1 show, that if $1 \leq n \leq 32$ and $n$ is odd, then $E_{z}(n) / R_{z}(n)=0.5$. This property is true for larger odd $n$ 's too.

Lemma 8 If $1 \leq k \leq 600$, then

$$
\frac{E_{z}(2 k-1)}{R_{z}(2 k-1)}=0.5
$$

Proof. See the computed values of $R_{z}(n)$ and $E_{z}(n)$ in [68].

Table 2 contains the ratios $E_{z}(n) / G_{z}(n)$ for $n=23, \ldots, 29$ and the ratios $T(n) / G_{z}(n)$ for $n=30$ and $n=31$.

The data in Table 2 show that the function $G_{z} / E_{z}$ ) is decreasing. We suppose that this function tends monotonically decreasing to zero when $n$ tends to infinity (in a similar way as the function $G(n) / E(n)$ tends to zero according to Corollary 23 [52, page 260].

Table 3 contains the values of $G_{z}(n), T(n)$, and $G_{z}(n) / T(n)$ for $n=30$ and $n=31$ : the ratio of the graphical and tested sequences is much higher and these ratios are increasing. These changes are dut to the fact that EGE2 jumps many nongraphical zerofree ebven sequences withous testing them.

\subsection{New algorithmic results}

Using the following Lemma 9 later we will further fasten EGE.

If $b=\left(b_{1}, \ldots, b_{n}\right)$ is a regular sequence, then $c=\left(c_{1}, \ldots, c_{n}\right)$ is called lexicographically $i$-smaller, than $b$ if there exist indices $i$ and $j$ such that

$$
1 \leq i<j<\leq n,
$$




\begin{tabular}{|r|r|r|r|}
\hline \hline $\mathrm{n}$ & $\mathrm{G}_{z}(\mathrm{n})$ & $\mathrm{E}_{z}(\mathrm{n})$ & $\mathrm{G}_{z}(\mathrm{n}) / \mathrm{E}_{z}(\mathrm{n})$ \\
\hline \hline 17 & 130038230 & 282861360 & 0.459724 \\
\hline 18 & 499753855 & 1101992870 & 0.453500 \\
\hline 19 & 1924912894 & 4298748300 & 0.447784 \\
\hline 20 & 7429160296 & 16789046494 & 0.442500 \\
\hline 21 & 28723877732 & 65641204200 & 0.437589 \\
\hline 22 & 111236423288 & 256895980068 & 0.433002 \\
\hline 23 & 431403470222 & 1006308200040 & 0.428699 \\
\hline 24 & 1675316535350 & 3945186233014 & 0.424648 \\
\hline 25 & 6513837679610 & 15478849767888 & 0.420821 \\
\hline 26 & 25354842100894 & 60774332618300 & 0.417197 \\
\hline 27 & 98794053269694 & 238775589937976 & 0.413752 \\
\hline 28 & 385312558571890 & 938702947395204 & 0.410473 \\
\hline 29 & 1504105116253904 & 3692471324505040 & 0.407344 \\
\hline 30 & 5876236938019300 & 14532512180224216 & 0.404351 \\
\hline 31 & 22974847474172100 & 57224797531384560 & 0.400148 \\
\hline
\end{tabular}

Table 2: The values of $G_{z}(n), E_{z}(n)$, and $G_{z}(n) / E_{z}(n)$ for $n=17, \ldots, 31$

\begin{tabular}{|r|r|r|r|}
\hline \hline $\mathrm{n}$ & $\mathrm{G}_{z}(\mathrm{n})$ & $\mathrm{T}(\mathrm{n})$ & $\mathrm{G}_{z}(\mathrm{n}) / \mathrm{T}(\mathrm{n})$ \\
\hline \hline 31 & 5876236938019300 & 6790865476867340 & 86,531487 \\
\hline 32 & 22974847471172100 & 26507499250791700 & 86,673010 \\
\hline
\end{tabular}

Table 3: The values of $G_{z}(n), T(n)$, and $G_{z}(n) / T(n)$ for $n=30$ and $n=31$

further

$$
c_{k}=b_{k} \quad \text { for } k=1, \ldots, i \text {, }
$$

and

$$
\sum_{k=i+1}^{n} c_{k} \leq \sum_{k=i+1}^{n} b_{k}
$$

Lemma 9 If $\mathrm{b}=\left(\mathrm{b}_{1}, \ldots, \mathrm{b}_{\mathrm{n}}\right)$ and is a nongraphical sequence and $\mathrm{c}=\left(\mathrm{c}_{1}, \ldots, \mathrm{c}_{\mathrm{n}}\right)$ is lexicographically $\boldsymbol{i}$-smaller than $\mathrm{b}$ for some $\mathrm{i}(1 \leq \mathfrak{i}<\mathrm{n}$, then $\mathrm{c}$ is also graphical.

Proof. See [54].

Using this lemma the running time of EGLJ decreased substantially. It was very useful when we enumerated the edge sequences of the simple graphs on 
30 vertices between June 21 and 18 July of 2013 and on 31 vertices between 18 July and 24 August (the results see in Table 4).

Using the results of Tripathi and Vijay [52, Lemma 6 and Theorem 7] we can substantially decrease the average testing time of the zerofree even sequences. It is known that the expected number of checking points proposed by Tripathi and Vijay is about $\mathfrak{n} / 2$ [52].

Algorithm EGE2 [51, pages 274-277] used in the enumerations for $n=30$ and $n=31$ vertices is based on Lemma 9 .

We develop algorithm EGE3 for the enumeration of the degree sequences in the case of 32 vertices. EGE3 will be based on Lemmas 10 and 11.

Lemma 10 If the investigated by EG3 sequence is graphical and has the form $\mathrm{b}=\mathrm{b}_{i_{1}}^{c_{1}} b_{i_{2}}^{c_{2}}$ and the upper bound $c_{1}\left(c_{1}-1\right)$ of the inner capacity of the $c_{1}$-length head of $\mathrm{b}$ covers $\mathrm{H}_{\mathrm{n}}$, that is if

$$
c_{1}\left(c_{1}-1\right) \geq H_{n}
$$

then all sequences starting with $\mathrm{b}_{1}^{\mathrm{c}_{1}}$ are graphical.

Proof. See [54].

The next lemma allows to enumerate many graphical sequences without their time consuming testing.

Lemma 11 If the investigated by EG3 graphical sequence has the form $\mathrm{b}=$ $b_{1}^{c_{1}} b_{2}^{c_{2}} \ldots b_{p}^{c_{p}} 1^{c_{p}+1}$, where $p \geq 1, b_{1}>b_{2}>\cdots>b_{p} \geq 3, c_{1}, \ldots, c_{p+1} \geq 1$, then all zerofree even sequences starting with the prefix

$$
b_{1}^{c_{1}} b_{2}^{c_{2}} \ldots b_{p-1}^{c_{p-1}} b_{p}^{c_{p}-1}\left(b_{p}-1\right)
$$

are also graphical.

Proof. See [54].

\subsection{New simulation results}

Table 4 contains the values of $G_{z}(n)$ and $G(n)$ for $n=1, \ldots, 31$. The values for $n=1, \ldots, 9$ were computed by E. Weisstein, for $n=10, \ldots, 20$ by G. Royle in 2006, for $n=21,22$ and $n=23$ by F. Ruskey in 2006, for $n=$ $24, \ldots, 29$ by T. Matuszka in January of 2013 , for $n=30$ by L. Lucz in July of 2013 and for $\mathrm{n}=31$ also by L. Lucz in September of 2013 [48, 50, 51, 52, 79].

Column 4 of Table 4 supports the following conjecture formulated by Gordon Royle in 2012. 
Conjecture 12 (Royle, 2012). If $\mathrm{n}$ tends to infinity, then $\mathrm{G}_{z}(\mathrm{n}+1) / \mathrm{G}_{z}(\mathrm{n})$ tends to 4 .

We think, that the following conjecture is also true.

Conjecture 13 If $\mathrm{n}$ tends to infinity, then $\mathrm{G}(\mathrm{n}+1) / \mathrm{G}(\mathrm{n})$ tends to 4 .

We observed that when we enumerated these sequences, that in the case $n=30$ vertices 85.40 percent, while in the case $n=31$ vertices 86.67 percent of the investigated potential degree sequences was graphical. Therefore it is useful if we know without a linear time testing that a given tested sequence is graphical.

Figure 1 shows the number of the tested and the graphical sequences as the function of the index of the slices when $n=30$.

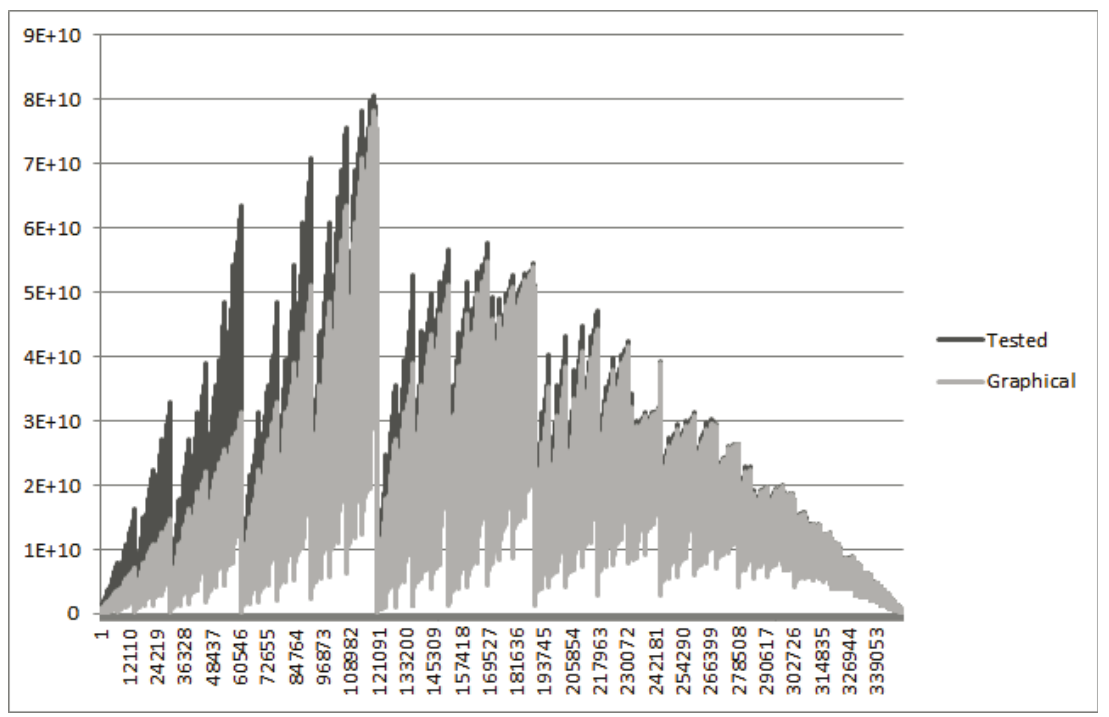

Figure 1: The number of tested (trimmed even) sequences and the number of graphical sequences as the function of the index of slices when $n=30$

Figure 2 shows the similar data for $\mathfrak{n}=31$.

We remark that on the site of the journal the Figures 1 and 2 are color (the graphical sequences are represented by red, while the tested sequences by blue color).

Table 5 contains the data of PC's used for the enumeration of $\mathrm{G}_{z}(31)$, where Comp. alg. $=$ Computer Algebra, Prog. lang. = Program languages, Core $=$ 


\begin{tabular}{||r|r|r|r|r|}
\hline \hline $\mathrm{n}$ & $\mathrm{G}_{z}(\mathrm{n})$ & $\mathrm{G}(\mathrm{n})$ & $\frac{\mathrm{G}_{z}(\mathrm{n}+1)}{\mathrm{G}_{z}(\mathrm{n})}$ & $\frac{\mathrm{G}(\mathrm{n}+1)}{\mathrm{G}(\mathrm{n})}$ \\
\hline \hline 1 & 0 & 1 & 0.000000 & 0.500000 \\
\hline 2 & 1 & 2 & 0.500000 & 0.500000 \\
\hline 3 & 2 & 4 & 3.500000 & 3.750000 \\
\hline 4 & 7 & 11 & 2.857143 & 2.818182 \\
\hline 5 & 20 & 31 & 3.550000 & 3.290323 \\
\hline 6 & 71 & 102 & 3.380282 & 3.352941 \\
\hline 7 & 240 & 342 & 3.629167 & 3.546784 \\
\hline 8 & 871 & 4361 & 3.614237 & 3.595218 \\
\hline 9 & 3148 & 16016 & 3.717889 & 3.6755544 \\
\hline 10 & 11655 & 59348 & 3.756323 & 3.742620 \\
\hline 11 & 43332 & 222117 & 3.773434 & 3.786674 \\
\hline 12 & 162769 & 836315 & 3.794439 & 3.802710 \\
\hline 13 & 614198 & 3166852 & 3.808465 & 3.817067 \\
\hline 14 & 2330537 & 12042620 & 3.822189 & 3.828918 \\
\hline 15 & 8875768 & 45967479 & 3.833125 & 3.839418 \\
\hline 16 & 33924859 & 176005709 & 3.843130 & 3.848517 \\
\hline 17 & 130038230 & 675759564 & 3.851172 & 3.856630 \\
\hline 18 & 499753855 & 2600672458 & 3.859479 & 3.863844 \\
\hline 19 & 1924912894 & 10029832754 & 3.866369 & 3.870343 \\
\hline 20 & 7429160296 & 38753710486 & 3.872612 & 3.876212 \\
\hline 21 & 28723877732 & 149990133774 & 3.878257 & 3.881553 \\
\hline 22 & 111236423288 & 581393603996 & 3.883410 & 3.886431 \\
\hline 23 & 431403470222 & 2256710139346 & 3.888124 & 3.890907 \\
\hline 24 & 1675316535350 & 8770547818956 & 3.894458 & 3.895031 \\
\hline 25 & 6513837,679610 & 34125389919850 & 3.895503 & 3.897978 \\
\hline 26 & 25354842100894 & 132919443189544 & 3.900159 & 3.898843 \\
\hline 27 & 98794053269694 & 518232001761434 & 3.903597 & 3.902238 \\
\hline 28 & 385312558571890 & & & \\
\hline 29 & 1504105116253904 & 2022337118015338 & 3.906814 & 3.905666 \\
\hline 30 & 5876236938019300 & 7898574056034638 & 3.909789 & 3.908734 \\
\hline 31 & 22974847474172100 & 30873429530206738 & --- & --- \\
\hline \hline
\end{tabular}

Table 4: The number $\mathrm{G}_{z}(\mathrm{n})$ of zerofree graphical sequences and the number $G(n)$ of graphical sequences for $n=1, \ldots, 31$, further the ratios $G_{z}(n) / G_{z}(n+$ 1 ) and $G(n) / G(n+1)$ for $n=1, \ldots, 30$

Core(TM)Kása $1=$ Z. Kása (Cluj), Kása $2=$ Z. Kása (Tg.-Mureś), Kása $3=$ Z. Kása // (Tg.-Mureś), Sp1 = Speed of a machine in GHz, Sp2 = Speed of the laboratory in GFLOPS, Intel $(\mathrm{R})=\operatorname{Intel}(\mathrm{R})$ Xeon $(\mathrm{R})$. 


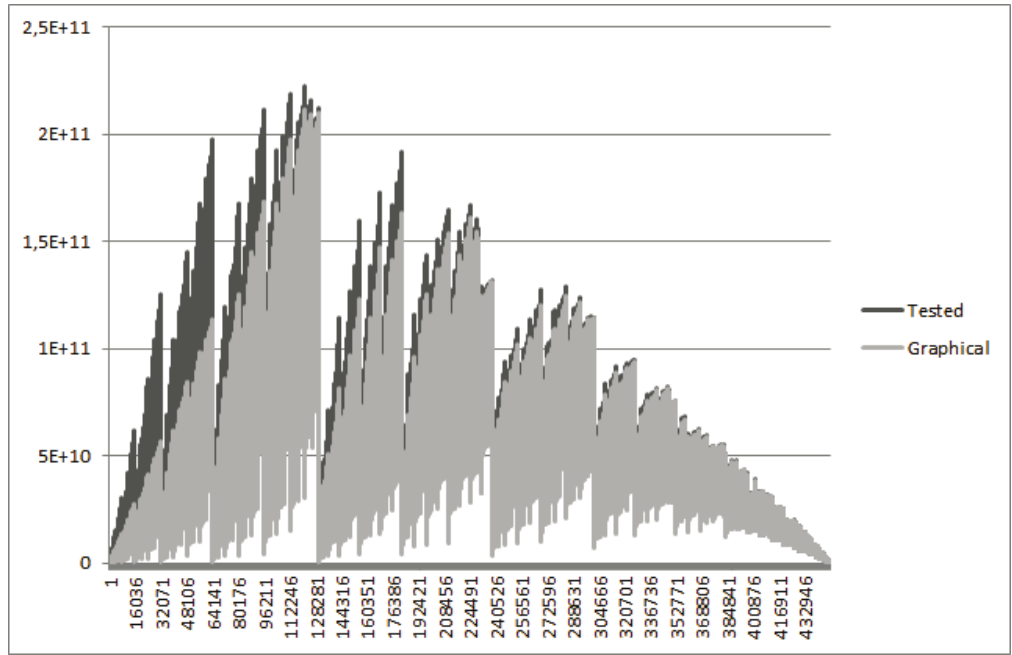

Figure 2: The number of tested (trimmed even) sequences and the number of graphical sequences as the function of the index of slices when $n=31$

The total number of machines was 350 .

Table 6 contains the algorithms, running times and number of jobs in the case $n=25, \ldots, 31$.

\subsection{The growth of the functions $R(n), E(n), R_{z}(n), E_{z}(n), G(n)$ and $\mathrm{G}_{z}(\mathrm{n})$}

In this subsection we present concrete values of the functions characterizing the sizes of the investigated sets of sequences.

The number $R(n)$ of the regular sequences is presented in Figure 1 of [52] for $n=1, \ldots, 38$ and up to $n=1200$ in [47].

The values of the zerofree regular $R_{z}(n)$ can be quickly computed using formula (22) in [47]. The values for $n=1, \ldots, 1200$ can be found in [68].

The number $E(n)$ of even sequences is presented in Figure 1 of [52] for $n=1, \ldots, 38$ and up to 1000 in [49] and up to $n=1200$ in [68].

The number $E_{z}(n)$ of the zerofree even sequences is contained in Figure 3 of [52] for $n=1, \ldots, 20$ (these data are the results of brute force simulation) and up to $\mathrm{n}=1200$ in [68].

The order of growth of these functions is $\Theta\left(4^{n} / n\right)$.

According to theorem of Burns $[22,52]$ the order of growth of $\mathrm{G}(\mathrm{n})$ is smaller (see 12). 


\begin{tabular}{||l|c|c|r|r||}
\hline Laboratory & Number & Type & Sp1 & Sp2 \\
\hline \hline Central & 87 & Core 2 Duo & 2.93 & 2041 \\
Comp. algebra & 13 & Core 2 Duo & 2.13 & 403 \\
Data base & 34 & Core 2 Duo & 3.25 & 1631 \\
Graphical & 16 & Core 2 Quad & 2.33 & 597 \\
Prog. lang. & 54 & Core 4 Duo & 3.25 & 6621 \\
PC1 & 20 & Core i5-2320 & 3.00 & 1920 \\
PC3 & 28 & Core i3-2100 & 3.10 & 1389 \\
PC4 & 19 & Core 2 Duo & 2.93 & 446 \\
PC5 & 19 & Core 4 Duo & 2.93 & 446 \\
PC6 & 18 & Core 2 Quad & 2,33 & 672 \\
PC7 & 18 & Core 2 Quad & 2.40 & 691 \\
PC9 & 19 & Core 2 Quad & 2.66 & 810 \\
Server & 1 & Core i5 650 & 3.20 & 26 \\
\hline Kása 1 & 1 & AMD K7 & 0.75 & 8 \\
Kása 2 & 1 & Intel (R) & 3.00 & 50 \\
Kása 3 & 1 & Core 4 Duo & 2.13 & 23 \\
P. Ősze & 350 & & & 17811 \\
\hline \hline Total & \multicolumn{3}{|l}{} \\
\hline \hline
\end{tabular}

Table 5: Names of laboratories, number of machines, type of machines, speed of machines in $\mathrm{GHz}$, speed of laboratories in GLOPS, used in the case $\mathfrak{n}=31$

The known values of $G(n)$ and $G_{z}(n)$ are summarized in Table 4.

\subsection{Further plans}

Our new program (EGE3) is able to jump the test of some part of zerofree graphical sequences [54]. Due to this property of the new program EGE3 the number of tested sequences is smaller than the number of zerofree graphical ones (see Table 7).

\section{Summary}

The log files and source codes of our programs can be found at http://people.inf.elte.hu/lulsaai/Holzhacker . 


\begin{tabular}{||c|r|r|r|r||}
\hline$n$ & Algorithm & $\begin{array}{r}\text { Running time } \\
\text { (in days) }\end{array}$ & $\begin{array}{r}\text { Running time } \\
\text { (in years) }\end{array}$ & Number of jobs \\
\hline \hline 25 & EGE & 26 & 0.0712 & 435 \\
26 & EGE & 70 & 0.1918 & 435 \\
27 & EGE & 316 & 0.8657 & 435 \\
28 & EGE & 1130 & 3.0959 & 2001 \\
29 & EGE & 6733 & 18.4466 & 15119 \\
\hline 30 & EGE2 & 7221 & 19.7835 & 351155 \\
\hline \hline 31 & EGE2 & 32702 & 89.5954 & 448957 \\
\hline \hline
\end{tabular}

Table 6: Number of vertices, used algorithm, total running time (in days and in years) and number of jobs

\begin{tabular}{|r|r|r|}
\hline $\mathrm{n}$ & $\mathrm{T}(\mathrm{n})$ & $\mathrm{G}_{z}(\mathrm{n})$ \\
\hline 3 & 3 & 2 \\
4 & 8 & 7 \\
5 & 24 & 20 \\
6 & 77 & 71 \\
7 & 245 & 240 \\
8 & 852 & 871 \\
9 & 2991 & 3148 \\
10 & 10807 & 11655 \\
11 & 39407 & 43332 \\
12 & 145673 & 162769 \\
13 & 542531 & 614198 \\
14 & 2036196 & 2330537 \\
15 & 7684164 & 8875768 \\
16 & 29143362 & 33924859 \\
17 & 110973050 & 130038230 \\
18 & 424055902 & 499753855 \\
19 & 1625265958 & 1924912894 \\
20 & 6245498873 & 7429160296 \\
\hline
\end{tabular}

Table 7: Number of vertices $(n)$, number of tested sequences $(T(n))$ and number of zerofree graphical sequences $\left(G_{z}(n)\right)$ 
Acknowledgements. The authors are indebted to Faculty of Informatics of Eötvös Loránd University for the possibility to run the server and client programs in its laboratories, further to Antal Sándor and Ferenc Saáry, Jr. (Eötvös Loránd University, Faculty of Informatics) for their technical help, for Bálint Csergő (Ulstream Hungary Kft.) for the conversion of the figures, for Zoltán Kása (Sapientia Hungarian University of Transylvania, Campus in Cluj and Tg.-Mureś) and for Péter Ösze (Ustream Hungary Kft.) for running the clients and Kristóf Szabados (Ericsson Hungary) for improving the jumping algorithm.

\section{References}

[1] S. R. Arikati, A. Maheshwari, Realizing degree sequences in parallel, SIAM J. Discrete Math. 9, 2 (1996) 317-338. $\Rightarrow 250$

[2] M. Ascher, Mu torere: an analysis of a Maori game, Math. Mag. 60, 2 (1987) 90-100. $\Rightarrow 248,251$

[3] T. M. Barnes, C. D. Savage, A recurrence for counting graphical partitions, Electron. J. Combin. 2 (1995), Research Paper 11, 10 pages (electronic). $\Rightarrow 252$

[4] T. M. Barnes, C. D. Savage, Efficient generation of graphical partitions, Discrete Appl. Math. 78, 1-3 (1997) 17-26. $\Rightarrow 252$

[5] M. D. Barrus, Hereditary unigraphs and Erdös-Gallai inequalities. arXiv:1302.2703v1 [mathCO] 12 February 2013, 23 pages. $\Rightarrow 250$

[6] M. D. Barrus, S. G. Hartke, K. F. Jao, D. B. West, Length threshold s for graphic lists given fixed largest and smallest entries and bounded gaps, Discrete Math. 312, 9 (2012) 1494-1501. $\Rightarrow 250$

[7] E. A. Bender, E. N. Canfield, The asymptotic number of labeled graphs with given degree sequences, J. Comb. Math. 24 (1978) 296-307. $\Rightarrow 250$

[8] C. Berge, Graphs and Hypergraphs, North Holland, 1973. $\Rightarrow 246,250$

[9] C. Berge, Graphs (third edition), North Holland, 2001 (first edition: 1989). $\Rightarrow$ 246,250

[10] A. Berger, Directed degree sequences, PhD Dissertation, Martin-LutherUniversität Halle-Wittenberg, 2011. http://wcms.uzi.uni-halle.de/ download $\cdot$ php?down=22851\&elem=2544689. $\Rightarrow 250$

[11] A. Berger, A note on the characterization of digraph sequences, arXiv, arXiv:1112.1215v1 [math.CO] (6 December 2011). $\Rightarrow 250$

[12] A. Berger, A note on the characterization of digraphic sequences, Discrete Math. 314 (2014) 38-41. $\Rightarrow 250$

[13] A. Berger, M. Müller-Hannemann, Uniform sampling of digraphs with a fixed degree sequence, in (ed. D. M. Thilikos) 36th Int. Workshop on Graph Theoretic Concepts in Computer Science (June 28 - 30, 2010, Zarós, Crete, Greece), LNCS 6410 (2010) 220-231. $\Rightarrow 250$ 
[14] A. Berger, M. Müller-Hannemann, Dag characterizations of directed degree sequences, Technical Report 2011/6 of University Halle-Wittenberg, Institute of Computer Science. $\Rightarrow 250$

[15] A. Berger, M. Müller-Hannemann, How to attack the NP-complete dag realization problems in practice. arXiv, arXiv:1203.36v1, 2012. http://arxiv.org/abs/1203.3636 $\Rightarrow 250$

[16] N. Bödei, Degree sequences of graphs (Hungarian), Mathematical master thesis (supervisor A. Frank), Dept. of Operation Research of Eötvös Loránd University, Budapest, 2010, 43 pages. $\Rightarrow 246$

[17] B. Bollobás, The distribution of the maximum degree of a random graph, Discrete Math. 32, 2 (1980) 201-203. $\Rightarrow 250$

[18] B. Bollobás, A probabilistic proof of an asymptotic formula for the number of labelled regular graphs, European J. Comb. 1, 4 (1980) 311-316. $\Rightarrow 250$

[19] B. Bollobás, Degree sequences of random graphs, Discrete Mathematics 33, 1 (1981) $1-19 . \Rightarrow 250$

[20] A. Brualdi, Matrices of zeros and ones with fixed row and column sum vectors, Linear Alg. Appl. 33 (1980) 159-231. $\Rightarrow 250$

[21] J. C. Brunson, The S-metric, the Beichl-Croteaux approximation and preferential attachment, arXiv:1308.4067v1 [mathCO] 19 August 2013. $\Rightarrow 250$

[22] J. M. Burns, The number of degree sequences of graphs, PhD Dissertation, MIT, 2007. $\Rightarrow 249,262$

[23] G. Cairns, S. Mendan, An improvement of a result of Zverovich-Zverovich, arXiv arXiv:1303.2144v1 [math.CO], 5 pages. $\Rightarrow 246$

[24] G. Cairns, S. Mendan, Degree sequences for graphs with loops, arXiv arXiv:1303.2145v1 [math.CO], 9 pages. $\Rightarrow 246$

[25] G. Cairns, S. Mendan, Y. Nikolayevsky, A sharp improvement of a result of Zverovich-Zverovich, arXiv arXiv:1310.3992v1 [math.CO], 7 pages. $\Rightarrow 246$

[26] S. A. Choudum, A simple proof of the Erdős-Gallai theorem on graph sequences, Bull. Austral. Math. Soc. 33 (1986) 67-70. $\Rightarrow 246$

[27] V. Chungphaisan, Conditions for sequences to be r-graphic, Discrete Math. 7 (1974) 31-39. $\Rightarrow 246$

[28] N. Cohen, Number of distinct degree sequences among all $n$-vertex graphs with no isolated vertices: new values for $n=20,21,22$, and 23, in: ed. by N. J. A. Sloane, The On-Line Encyclopedia of Integer Sequences, 2012, http://oeis.org/A095268. $\Rightarrow 246$

[29] P. Das, Characterization of unigraphic and unidigraphic integer-pair sequences, Characterization of unigraphic and unidigraphic integer-pair sequences Discrete Math. 37, 1 (1981) 51-66. $\Rightarrow 250$

[30] C. I. Del Genio, H. Kim, Z. Toroczkai, K. E. Bassler, Efficient and exact sampling of simple graphs with given arbitrary degree sequence, PLoS ONE 5, 4 e10012 (2010). $\Rightarrow 252$

[31] D. Dimitrov, Efficient computation of trees with minimal atom-bound connectivity index. arXiv:1305.1155v2 [csDM] 4 October 2013. $\Rightarrow 250$ 
[32] P. Erdős, T. Gallai, Graphs with vertices having prescribed degrees (Hungarian), Mat. Lapok 11 (1960) 264-274. $\Rightarrow 246,250,251$

[33] P. Erdős, L. B. Richmond, On graphical partitions, Combinatorica 13, 1 (1993) $57-63 . \Rightarrow 252$

[34] P. L. Erdős, S. Z. Kiss, I. Miklós, On the swap-distances of different realizations of a graphical degree sequence, Comb. Probab. Comp. 22, 3 (2013) 366-383. $\Rightarrow$ 250

[35] P. L. Erdős, Z. Király, I. Miklós, L. Soukup, Constructive sampling and counting graphical realizations of restricted degree sequences, arXiv arXiv:13017523v3 [math.CO], 24 pages. $\Rightarrow 250$

[36] P. L. Erdős, I. Miklós, Z. Toroczkai, A simple Havel-Hakimi type algorithm to realize graphical degree sequences of directed graphs, Electron. J. Combin. 17, 1 (2010) R66, 10 pages. $\Rightarrow 250$

[37] P. L. Erdős, I. Miklós, Z. Toroczkai, A decomposition based proof for fast mixing of a Markov chain over balanced realizations of a joint degree matrix, arXiv, arXiv:1307.5295v1 [math.CO], 2013, 18 pages. $\Rightarrow 250$

[38] C. Greenhill, A polynomial bound on the mixing time of a Markov chain for sampling regular directed graphs, Electron. J. Combin. 18 \&P234, 2011, 49 pages. $\Rightarrow 250$

[39] S. L. Hakimi, On the realizability of a set of integers as degrees of the vertices of a simple graph, J. SIAM Appl. Math. 10 (1962) 496-506. $\Rightarrow 246,250$

[40] S. L. Hakimi, On the degrees of the vertices of a graph, F. Franklin Institute 279, 4 (1965) 290-308. $\Rightarrow 250$

[41] F. Harary, E. M. Palmer, Graphical Enumeration, Academic Press, New York and London, 1973. $\Rightarrow 250$

[42] V. Havel, A remark on the existence of finite graphs (Czech), C̆asopis Pĕst. Mat. 80 (1955) 477-480. $\Rightarrow 246,250$

[43] P. Hell, D. Kirkpatrick, Linear-time certifying algorithms for near-graphical sequences. Discrete Math. 309, 18 (2009) 5703-5713. $\Rightarrow 246$

[44] A. Iványi, Reconstruction of complete interval tournaments, Acta Univ. Sapientiae, Inform. 1, 1 (2009) 71-88. $\Rightarrow 246,252$

[45] A. Iványi, Reconstruction of complete interval tournaments. II, Acta Univ. Sapientiae, Math. 2, 1 (2010) 47-71. $\Rightarrow 246,252$

[46] A. Iványi, Degree sequences of multigraphs. Annales Univ. Sci. Budapest., Sect. Comp. 37 (2012) 195-214. $\Rightarrow 246,250,252$

[47] A. Iványi, L. Lucz, G. Gombos, T. Matuszka C $(2 n+1, n+1)$ : number of ways to put $n+1$ indistinguishable balls into $n+1$ distinguishable boxes $=$ number of $(n+1)$-st degree monomials in $n+1$ variables $=$ number of monotone maps from $1 \ldots \mathrm{n}+1$ to $1 \ldots \mathrm{n}+1$, in (ed. N. J. A. Sloane): The On-Line Encyclopedia of the Integer Sequences. 2013. http://oeis.org/A001700. $\Rightarrow 247,262$

[48] A. Iványi, L. Lucz, G. Gombos, T. Matuszka The number of degree-vectors for simple graph. in (ed. N. J. A. Sloane): The On-Line Encyclopedia of the Integer Sequences. 2013. http://oeis.org/A004251. $\Rightarrow 246,251,259$ 
[49] A. Iványi, L. Lucz, G. Gombos, T. Matuszka, Number of bracelets (turn over necklaces) with $n$ red, 1 pink and $n-1$ blue beads; also reversible strings with $n$ red and $n-1$ blue beads, in (ed. by N. J. A. Sloane), The On-Line Encyclopedia of Integer Sequences, 2013. http://oeis.org/A005654. $\Rightarrow 262$

[50] A. Iványi, L. Lucz, G. Gombos, T. Matuszka, Number of distinct degree sequences among all n-vertex graphs with no isolated vertices: new values for $\mathrm{n}=24,25,26,27,28$, and 29, in (ed. by N. J. A. Sloane): The On-Line Encyclopedia of Integer Sequences, 2013, http://oeis.org/A095268. $\Rightarrow 247,259$

[51] A. Iványi, L. Lucz, T. Matuszka, S. Pirzada, Parallel enumeration of degree sequences of simple graphs. Acta Univ. Sapientiae, Inform. 4, 2 (2012) 260-288. $\Rightarrow 247,248,249,252,259$

[52] A. Iványi, L. Lucz, T. F. Móri, P. Sótér, On the Erdős-Gallai and Havel-Hakimi algorithms. Acta Univ. Sapientiae, Inform. 3, 2 (2011) 230-268. $\Rightarrow 245,246,247$, 248, 249, 250, 251, 252, 257, 259, 262

[53] A. Iványi, S. Pirzada, Comparison based ranking, in: Algorithms of Informatics, Vol. 3 (ed. A. Iványi), AnTonCom, Budapest 2011, 1209-1258. $\Rightarrow 246$

[54] A. Iványi, K. Szabados, Parallel enumeration of degree sequences (Hungarian). Alk. Mat. Lapok, 40 pages, submitted. $\Rightarrow 258,259,263$

[55] R. H. Johnson, properties of unique realizations - a survey, Discrete Math. 3, 1 (1980) $185-192 . \Rightarrow 250$

[56] J. H. Kim, B. Piztttel, Confirming the Kleitman-Winston conjecture on the largest coefficient in a q-Catalan number, J. Comb. Theory A 99, 2 (2000) 19-206. $\Rightarrow 250$

[57] H. Kim, Z. Toroczkai, I. Miklós, P. L. Erdős, L. A. Székely: Degree-based graph construction, J. Physics: Math. Theor. A 42 (2009), 392001, 10 pages. $\Rightarrow$

[58] Z. Király, Recognizing graphic degree sequences and generating all realizations. Egres Technical Reports, TR-2011-11 April 23, 2012, 12 pages. $\Rightarrow 246,250$

[59] D. J. Kleitman, D. Wang, Algorithms for constructing graphs and digraphs with given valences and factors, Discrete Math. 6 (1973) 79-88. $\Rightarrow 250$

[60] D. J. Kleitman, K. J. Winston, Forests and score vectors. Combinatorica 1 (1981) $49-51 . \Rightarrow 250$

[61] D. E. Knuth, The Art of Computer Programming, Volume 4A, Addison Wesley, 2011. $\Rightarrow 246$

[62] M. Koren, Sequences with a unique realization by simple graphs, J. Comb. Theory, Ser. B 21, 3 (1976) 235-244. $\Rightarrow 250$

[63] M. D. LaMar, Algorithms for realizing degree sequences of directed graphs. arXiv, 2010. http://arxiv.org/abs/0906.0343. $\Rightarrow 246$

[64] V. Librandi, Number of bracelets (turn over necklaces) with $n$ red, 1 pink and $\mathrm{n}-1$ blue beads for $\mathrm{n}=1, \ldots, 1000$, in (ed. N. J. A. Sloane): The On-Line Encyclopedia of the Integer Sequences. 2012. http://oeis .org/A005654/b005654 . txt $\Rightarrow 248,252$ 
[65] X. Lu, S. Bressan, Generating random graph sequences, in (ed. J. X. Yu, M. H. Kim, R. Unland): DASFAA2011, Part I, LNCS 6587, Springer-Verlag, 2011, $570-579 . \Rightarrow 252$

[66] L. Lucz, Analysis of degree sequences of graphs (Hungarian), Student thesis awarded by first prize in the Hungarian Scientific Student Conference, Budapest, 2013. Eötvös Loránd University, Faculty of Informatics, Budapest, 2011. http://people.inf.elte.hu/lulsaai/diploma. $\Rightarrow 252$

[67] B. D. McKay, X. Wang, Asymptotic enumeration of tournaments with a given score sequence. J. Comb. Theory A, 73, (1) (1996) 77-90. $\Rightarrow$

[68] T. Matuszka, Programs and Results Connected with Degree Sequences. ELTE IK, Budapest, 2013. $\Rightarrow 247,248,257,262$

[69] D. Meierling, L. Volkmann, A remark on degree sequences of multigraphs, Math. Methods Operation Research, 69, 2 (2009) 369-374. $\Rightarrow 246$

[70] J. W. Miller, Reduced criteria for degree sequences, Discrete Math. 313, 4 (2013) $550-562 . \Rightarrow 246$

[71] R. Milo, N. Kashtan, S. Itzkovitz, M.E.J. Newman, U. Alon, On the uniform generation of random graphs with prescribed degree sequences, arXiv, arXiv:condmat/0312028v2 [cond-mat.stat-mech], 2004, 4 pages. $\Rightarrow 250$

[72] Miklós, J. Podani, Randomization of presence-absence matrices: comments and new algorithms, Ecology, 85, 1 (2004) 86-92. $\Rightarrow 250$

[73] T. D. Noe, Table of $\mathrm{a}(\mathrm{n})$ for $\mathrm{n}=1, \ldots, 100$, in (ed. N. J. A. Sloane): The OnLine Encyclopedia of the Integer Sequences. 2010. http://oeis .org/A001700. $\Rightarrow$ 252

[74] T. D. Noe, Table of binomial coefficients $\mathrm{C}(2 \mathrm{n}-1, \mathrm{n})$, in (ed. N. J. A. Sloane): The On-Line Encyclopedia of the Integer Sequences. 2012, http://oeis.org/ A001791. $\Rightarrow 252$

[75] S. Özkan, Generalization of the Erdős-Gallai inequality. Ars Combin., 98 (2011) $295-302 . \Rightarrow 246$

[76] A. N. Patrinos, S. L. Hakimi, Relations between graphs and integer-pair sequences. Discrete Math., 15, 4 (1976) 347-358 $\Rightarrow 250$

[77] S. Pirzada, Introduction to Graph Theory, Universities Press (India) Private Limited, 2012. $\Rightarrow 246$

[78] R. C. Read, The enumeration of locally restricted graphs (I). J. London Math. Soc., 34 (1959) 417-436. $\Rightarrow 250$

[79] G. Royle, Is it true that $a(n+1) / a(n)$ tends to 4? In (ed. N. J. A. Sloane): The On-Line Encyclopedia of the Integer Sequences. 2012. http://oeis.org/A095268 $\Rightarrow 259$

[80] F. Ruskey, Number of distinct degree sequences among all $n$-vertex graphs with no isolated vertices for $\mathrm{n}=21,22$ and 23, in (ed. N. J. A. Sloane): The On-Line Encyclopedia of the Integer Sequences. 2006. http://oeis.org/A095268. $\Rightarrow 247$

[81] H. J. Ryser, Matrices of zeros and ones. Bull. of Amer. Math. Soc. 66, 6 (1960) $442-464 . \Rightarrow 250$

[82] R. L. Shuo-Yen, Graphic sequences with unique realization, J. Comb. Theory, Ser. B, 19 (1975) 42-68. $\Rightarrow 250$ 
[83] G. Sierksma, H. Hoogeveen, Seven criteria for integer sequences being graphic, J. Graph Theory 15, (2) (1991) 223-231. $\Rightarrow 246$

[84] N. J. A. Sloane, The number of degree-vectors for simple graphs, in (ed. N. J. A. Sloane): The On-Line Encyclopedia of the Integer Sequences. 2011. http: //oeis.org/A004251. $\Rightarrow 251$

[85] N. J. A. Sloane, S. Plouffe, The Encyclopedia of Integer Sequences, Academic Press, 1995. $\Rightarrow 248$

[86] M. Takahashi, Optimization Methods for Graphical Degree Sequence Problems and their Extensions, $\mathrm{PhD}$ thesis, Graduate School of Information, Production and systems, Waseda University, Tokyo, 2007. http://hdl.handle.net/2065/28387. $\Rightarrow 246$

[87] A. Tripathi, H. Tyagy, A simple criterion on degree sequences of graphs. Discrete Appl. Math., 156, 18 (2008) 3513-3517. $\Rightarrow 246$

[88] A. Tripathi, S. Vijay, A note on a theorem of Erdős \& Gallai. Discrete Math., 265, 1-3 (2003) 417-420. $\Rightarrow 246$

[89] A. Tripathi, S. Venugopalan, D. B. West, A short constructive proof of the ErdősGallai characterization of graphic lists. Discrete Math., 310, 4 (2010) 833-834. $\Rightarrow 246$

[90] R. I. Tyshkevich, O. I. Melnikov, V. M. Kotov, On graphs and degree sequences: a canonical decomposition (Russian), Kibernetika 6 (1981) 5-8. $\Rightarrow 246$

[91] K. J. Winston, D. J. Kleitman, On the asymptotic number of tournament score sequences. J. Comb. Theory A 35, 2 (1983) 208-230. $\Rightarrow 250$

[92] I. E. Zverovich, V. E. Zverovich, Contributions to the theory of graphic sequences, Discrete Math., 105, 1-3 (1992) 293-303. $\Rightarrow 246$

Received: August 10, 2013 • Revised: December 30, 2013 